\title{
Digital crowdsourced intervention to promote HIV testing among MSM in China: study protocol for a cluster randomized controlled trial
}

Ci Ren ${ }^{1 \dagger}$, Joseph D. Tucker ${ }^{2+}$, Weiming Tang ${ }^{2+}$, Xiaorun Tao ${ }^{3}$, Meizhen Liao ${ }^{3}$, Guoyong Wang ${ }^{3}$, Kedi Jiao ${ }^{1}$, Zece Xu', Zhe Zhao', Yu Yan', Yuxi Lin', Chuanxi Li', Lin Wang ${ }^{1}$, Yijun Li', Dianmin Kang ${ }^{3 *}$ and Wei Ma ${ }^{1 *}$

\begin{abstract}
Background: Men who have sex with men (MSM) are an important HIV key population in China. However, HIV testing rates among MSM remain suboptimal. Digital crowdsourced media interventions may be a useful tool to reach this marginalized population. We define digital crowdsourced media as using social media, mobile phone applications, Internet, or other digital approaches to disseminate messages developed from crowdsourcing contests. The proposed cluster randomized controlled trial (RCT) study aims to assess the effectiveness of a digital crowdsourced intervention to increase HIV testing uptake and decrease risky sexual behaviors among Chinese MSM.

Methods: A two-arm, cluster-randomized controlled trial will be implemented in eleven cities (ten clusters) in Shandong Province, China. Targeted study participants will be 250 MSM per arm and 50 participants per cluster. MSM who are 18 years old or above, live in the study city, have not been tested for HIV in the past 3 months, are not living with HIV or have never been tested for HIV, and are willing to provide informed consent will be enrolled. Participants will be recruited through banner advertisements on Blued, the largest gay dating app in China, and inperson at community-based organizations (CBOs). The intervention includes a series of crowdsourced intervention materials (24 images and four short videos about HIV testing and safe sexual behaviors) and HIV self-test services provided by the study team. The intervention was developed through a series of participatory crowdsourcing contests before this study. The self-test kits will be sent to the participants in the intervention group at the 2 nd and 3 rd follow-ups. Participants will be followed up quarterly during the 12-month period. The primary outcome will be self-reported HIV testing uptake at 12 months. Secondary outcomes will include changes in condomless sex, selftest efficacy, social network engagement, HIV testing social norms, and testing stigma.

(Continued on next page)
\end{abstract}

\footnotetext{
* Correspondence: dianminkang66@163.com; weima@sdu.edu.cn

${ }^{\dagger}$ Ci Ren, Joseph D. Tucker and Weiming Tang contributed equally to this work.

${ }^{3}$ Institution for AIDS/STD Control and Prevention, Shandong Center for Disease Control and Prevention, Jinan 250014, Shandong, China

'Department of Epidemiology, School of Public Health, Cheeloo College of Medicine, Shandong University, Jinan 250012, Shandong, China

Full list of author information is available at the end of the article
}

(c) The Author(s). 2020 Open Access This article is licensed under a Creative Commons Attribution 4.0 International License, which permits use, sharing, adaptation, distribution and reproduction in any medium or format, as long as you give appropriate credit to the original author(s) and the source, provide a link to the Creative Commons licence, and indicate if changes were made. The images or other third party material in this article are included in the article's Creative Commons licence, unless indicated otherwise in a credit line to the material. If material is not included in the article's Creative Commons licence and your intended use is not permitted by statutory regulation or exceeds the permitted use, you will need to obtain permission directly from the copyright holder. To view a copy of this licence, visit http://creativecommons.org/licenses/by/4.0/ The Creative Commons Public Domain Dedication waiver (http://creativecommons.org/publicdomain/zero/1.0/) applies to the data made available in this article, unless otherwise stated in a credit line to the data. 
(Continued from previous page)

Discussion: Innovative approaches to HIV testing among marginalized population are urgently needed. Through this cluster randomized controlled trial, we will evaluate the effectiveness of a digital crowdsourced intervention, improving HIV testing uptake among MSM and providing a resource in related public health fields.

Trial registration: ChiCTR1900024350. Registered on 6 July 2019.

Keywords: HIV testing, Men who have sex with men (MSM), Digital, Crowdsourced intervention, Clusterrandomized controlled trial, China

\section{Background}

Chinese MSM still have a high burden of incident HIV infection, suggesting the need for enhanced HIV control strategies. As of October 2019, MSM accounted for $23.0 \%$ of newly reported HIV cases in China, increased from 14.7\% of new HIV cases in 2011 [1, 2]. Although the Chinese clinical and public health systems have organized many free HIV testing programs, two systematic reviews show that the HIV testing rate among MSM in China remains low [3, 4]. In order to improve the HIV testing rate and reach the first 90 target of the 90-90-90 UNAIDS goals [5], innovative approaches are urgently needed. Digital crowdsourced media may be a useful tool. We define digital as social media, mobile phone applications, Internet, or other approaches that use digital connections.

Digital approaches have been used to promote HIV prevention, especially for key populations like MSM [69]. Digital approaches can reach remote participants, limit stigma, provide a platform for anonymity and privacy, and increase community engagement [7, 9-11]. Previous surveys suggested that digital interventions can promote HIV testing and safe sexual behaviors through providing information related to HIV, offering HIV testing services, collecting different creative ideas to design intervention and linking people to the community [10, 12]. In China, the Internet has become a major way to find gay partners among MSM [13, 14]. A crosssectional survey of 61 cities in China showed that $73.5 \%$ of MSM had found recent sex partners online [15], providing a strong foundation for digital interventions. According to the data of MSM national sentinel surveillance in China, only $26.3 \%$ men had received an HIV test and know the results in the last year [16]. The high rates of Internet use and low HIV testing rate among MSM in China make digital approaches a useful tool in reaching high-risk populations and building low-cost HIV prevention interventions to promote HIV testing [17, 18].

Crowdsourcing provides an opportunity to develop HIV testing services tailored to meet local community needs while $[19,20]$. Crowdsourcing has a group of experts and non-experts working together to solve a common problem and sharing solutions with the public [19, 21]. Crowdsourcing may be useful in health research and has been used in cancer, malaria, and HIV research [22-25]. It has several advantages. First, crowdsourcing provides a platform for the people who have no formal training to make a contribute and enhance community engagement [20, 26-28]. Second, crowdsourcing approaches have been found effective in many trials [24, 29]. Third, crowdsourcing can help to access vulnerable groups that would otherwise be difficult to engage [27, 30]. As a result, crowdsourcing also represents a relatively low-cost method to collect information from a large number of people [20, 24, 28, 31]. However, most crowdsourced interventions have been conducted in large cities with few in smaller cities and less developed areas [29, 32]. Digital crowdsourced media may be a good way to promote HIV testing and healthy sexual behaviors in rural or remote regions.

This study proposal aims to describe the designing of the cluster RCT and to evaluate the efficacy of digital crowdsourced intervention to improve HIV testing uptake among MSM in China.

\section{Trial aims}

The first aim of this trial is to improve HIV testing uptake among MSM in eleven target cities. The study will evaluate the effectiveness of a digital crowdsourced intervention compared to current HIV interventions organized by local centers for disease control and prevention (CDCs). We hypothesize that the digital crowdsourced intervention will be superior in improving HIV testing uptake. The second aim is to compare secondary outcomes (including condom use, social networking, HIV stigma, and others) between the digital crowdsourced intervention arm and the current HIV intervention. We hypothesize that the digital crowdsourced intervention will be superior in promoting safe sexual behaviors.

\section{Methods \\ Design}

As the intervention in our study will involve two levelsthe community level and the individual level, and MSM in the same city often communicate with each other, we adopt a cluster-randomized controlled trial with one intervention arm and one control arm (Fig. 1). The 
Select target cities in Shandong Province ( $\mathrm{N}=11)$

- With CDC MSM sentinel surveillance site

- Capacity for recruiting adequate MSM

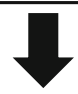

\section{Block Design (1:1 ratio)}

- Stratified by the city's number of HIV-positive in 2018, average GDP in 2017, cumulative number of HIV-positive from 2012-2018 and year's population at the end of 2017

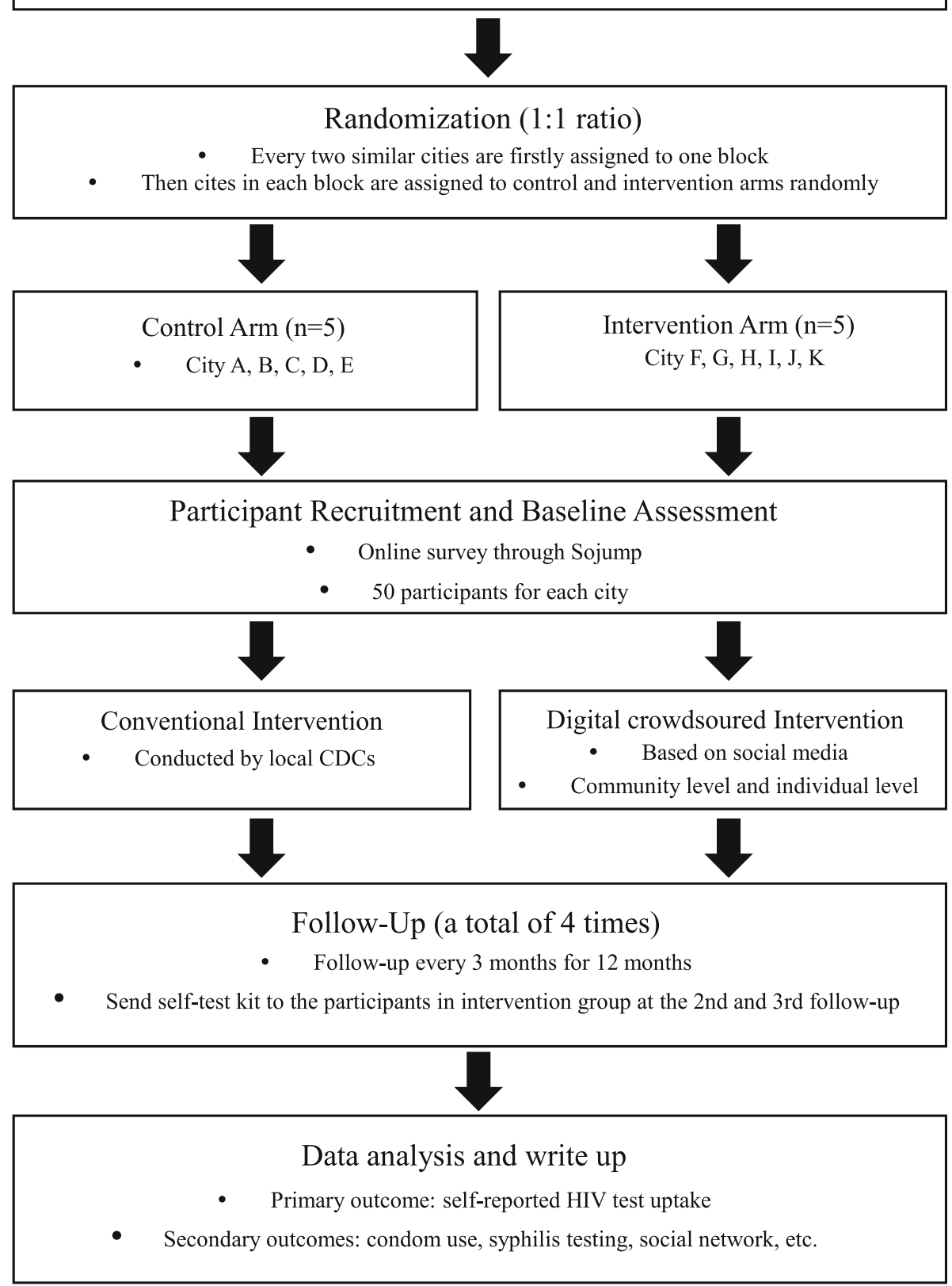

Fig. 1 Schematic of crowdsourced intervention implementation 
cluster unit will be the city. Surveys will be conducted at baseline and every 3 months thereafter (Fig. 2). A total of ten clusters, including City A, City B, City C, City D, City E, City F, City G, City H, City I, City J, and City K from Shandong Province, were chosen based on the following criteria: (1) CDC MSM sentinel surveillance sites and (2) capacity for recruiting sufficient MSM. City J and a nearby City $\mathrm{K}$ are in the same cluster because the number of estimated MSM in both cities is small.

\section{Randomization}

We randomly assigned cities in each block (1:1) to either the intervention group or the control group with a block design, stratified by the city's number of people living with HIV in 2018, average GDP in 2017, cumulative number of HIV-positive from 2012 to 2018 and population at the end of 2017. We have a total of five blocks involving ten clusters: Block 1 consists of City A and City F; Block 2 consists of City B and City G; Block 3 consists of City C, City J, and City K; Block 4 consists of City D and City H; and Block 5 consists of City E and City I. Then, the ten clusters were randomized with five
(City A, City B, City C, City D, City E) in the intervention arm and five (City F, City G, City H, City I, City J, City $\mathrm{K}$ ) in the control arm. Random numbers were generated using SAS 9.4 software. Cities in the intervention arm will implement the digital crowdsourced intervention while cities in the control arm will implement conventional interventions organized by local CDCs. Participants and data analysts will be blinded in this trial.

\section{Participants and eligibility criteria}

Eligibility criteria for participants were as follows: (1) aged at least 18 years, (2) had anal sex with men in the past 1 year, (3) currently living and planning to live in the city for the next 12 months, (4) born biologically male, (5) agree to provide phone number (just for follow up), (6) willing to participate in the follow-up survey every 3 months, and (7) fully understand the facts about the trial with the informed consent. The informed consent form will be shown to the potential participants at the beginning of the online questionnaire. Only those who clicked the "agree" option may be included in the

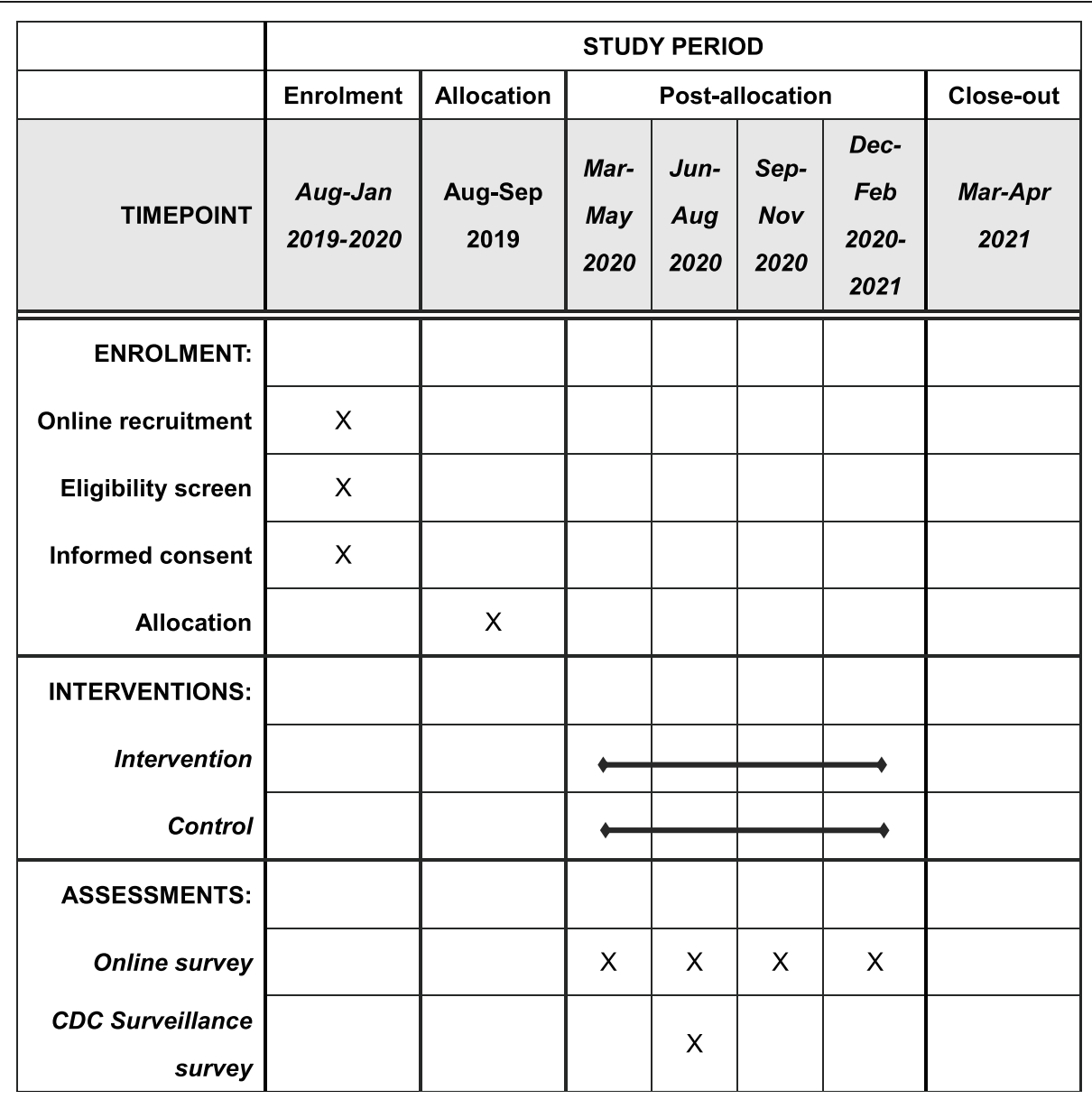

Fig. 2 SPIRIT figure of study timeline 
study. Participants living with HIV will be invited to complete the baseline survey but will not be followed.

\section{Study measures and recruitment}

We have built an online questionnaire (see Additional file 1) using Sojump Survey Software. The questionnaire is divided into two parts. Part 1 includes the informed consent form and six items related to study eligibility. Part 2 includes the main items related to this trial. MSM who are determined eligible in the first part proceed to the second section. They will be required to add us as contacts on WeChat (the largest social mobile phone app in China) so that we can establish an online cohort. Participants who do not meet all requirements can scan the QR codes to get 0.14USD (1RMB) on WeChat as an inducement. MSM will be recruited through banner advertisements on Blued, the largest gay dating app in China. CBOs in each study city will also assist with recruitment. In addition to direct recruitment through digital approaches, eligible participants will be invited to refer no more than five friends from their social networks of MSM and can receive a 1.44USD (10RMB) per person as incentive if their friends meet the requirements of this trial. All participants who enroll in the trial will receive a 7.22USD (50RMB) incentive for baseline survey and 7.22USD (50RMB) for each followup. Surveys will be conducted at baseline and every 3 months thereafter. Those who complete all five surveys will have an opportunity to win an iPad mini.

All the information in the questionnaire is selfreported. The questionnaire used in MSM surveillance sites run by local $\mathrm{CDCs}$ in each city will include additional questions about sexual partners, social media engagement, HIV/syphilis testing, and HIV/syphilis test results (see Additional file 2). Cell phone numbers will be used to link CDC and online survey data sets so the self-report data can be triangulated with CDC surveillance data during the same period.

\section{Intervention}

The intervention includes 24 images and 4 short videos about HIV testing and safe sexual behaviors. We will also send self-test kits to the participants in intervention group with their consent to evaluate the effectiveness of self-test kit in improving HIV testing rate at the 2nd and 3rd follow-ups. Images and videos were developed through a series of participatory crowdsourcing contests before this study. These included a sprint-like designathon that identified digital HIV self-testing as a priority [33] and an open call for images and videos [29]. The 24 images aim to address the importance of HIV testing to improve HIV testing uptake. The purpose of the 4 short videos of $50 \mathrm{~s}$ to $1 \mathrm{~min}$ in duration is to enhance the awareness of safe sexual behaviors like condom use among MSM and encourage them to take regular HIV testing. The images and videos can be found in Additional file 3. The intervention will be implemented at the individual level through "WeChat", and at community level through WeChat-group and WeChat-moments (a function on WeChat that people can share their life with friends).

The intervention will be implemented in five clusters (City F, City G, City H, City I, City J, City K) in the intervention arm. For individual-level intervention, the images will first be shown at the end of the baseline questionnaire, and then, we will send one image every 2 weeks and one video every 3 months to the participants via WeChat. We will also send self-test kits to the participants in the intervention group at the 2nd and 3rd follow-ups. As part of the community-level intervention, we will set up several WeChat groups in each city and invite 30-40 participants in each group. Messages about HIV testing and safe sexual behavior from authoritative facilities (hospital, CDCs, CBOs, etc.) will be distributed to these groups and WeChat-moments every 2 weeks. We will encourage members in these groups to talk about HIV through these private groups. We will invite volunteers from local $\mathrm{CBOs}$ to join the groups. Team members of this study will participate in discussion if the participants have any question. Participants in other five cities (City A, City B, City C, City D, City E) will receive routine intervention by local CDCs. All concomitant care is acceptable with no restrictions on what participants may seek.

\section{Follow-up}

All participants will receive an online survey at baseline and follow-up surveys will be conducted every 3 months. Each man will participate in a total of five surveys. At the 2nd and 3rd follow-ups, we will send self-test kits to the participants in the intervention group to evaluate the effectiveness of self-test kit in improving HIV testing rate.

\section{Outcomes}

The primary outcome of this study will be the uptake of self-reported HIV testing uptake after 12 months. We have set ten monitoring sentinels in the eleven cities so that the self-reported HIV testing rate can be triangulated with the rate from surveillance data during the same period. In addition, participants who receive the self-test kit are required to send their results to us on WeChat so that we can verify that the self-reported results are consistent with the self-test results. An increase of $20 \%$ in testing rate (assuming a proportion of HIV testing of $70 \%$ in intervention arm and $50 \%$ in control arm) was chosen as the superiority margin. This choice was based on the data of MSM sentinel surveillance 
collected by CDCs of Shandong Province in last 1 year. Secondary outcomes include condom use, syphilis testing, social network characteristics, and others (see Additional file 4).

\section{Sample size}

A two-arm, cluster-randomized controlled trial will be used. The sample size was calculated based on the primary outcome. We assumed that a digital crowdsourced intervention will be superior to CDC routine intervention in promoting HIV testing rate among MSM who have not been tested in the past 3 months. Assuming the HIV testing rate is $70 \%$ in intervention cities and $50 \%$ in the control cities, a total of ten clusters (five in intervention group and five in control group), an intraclass correlation coefficient of 0.02 (usually between 0.01 to 0.03 ), two-sided $\alpha=0.05$, power $=0.85$, and the loss of follow-up is $30 \%$, the total sample size is 500 men (50 for each cluster). The calculation was made using the software PASS 15.

\section{Timeline}

This study will last 17 months. The first 5 months will be the baseline survey. Given the total population of MSM are small in some cities, we wanted to ensure sufficient time for participant recruitment. CDC surveillance surveys will also be conducted in participating cities. The following 12 months will be the intervention phase. The intervention will be implemented in City F, City G, City H, City I, City J, and City K following the cluster RCT design outlined in Fig.2. All the participants in the eleven cities will be surveyed at baseline and every 3 months thereafter. In the 17 th month, the last followup survey will be implemented.

\section{Data collection and management}

The Sojump Survey Software will be used for data collection and storage. In this study, we will collect information about socio-demographics, sexual behaviors, HIV and syphilis testing behaviors, social network characteristics, and psychological characteristics through an online questionnaire. The online tool and questionnaire were used in our original stepped wedge cluster randomized controlled trial [29]. Socio-demographic characteristics include age, occupation, education level, annual income, marital status, sexual orientation, and sexual orientation disclosure. Behavioral variables consist of sex partners, frequency of sex, condom use, self-reported HIV testing (including both facility-based and selftesting), syphilis testing, and social networking. Psychological characteristics include HIV testing self-efficacy and HIV stigma.

To improve data quality, we have set up a verification mechanism. When a questionnaire has been completed, we will check it first. If it has only one logical error, we will verify with the participant. When there is more than one logical error in the questionnaire, the participant will no longer be included in the study. All questions in our online survey have been set as compulsory questions, which can improve data completeness.

Final survey data completed by all participants will be saved in a secure computer only used for saving data, discarding other privacy issues such as open network environments. Passwords and firewalls will also be used to protect the data.

An internal advisory committee composed of STI experts has been established. The committee will guide us in study design and data analysis and meet regularly to review and assess progress in data collection and research.

\section{Analysis}

The socio-demographic characteristics will be analyzed using descriptive statistics. Comparison in baseline characteristics between the intervention and control arm will be conducted using a chi-squared test adjusted for clustering. The primary outcome of this trial will be the uptake of self-reported HIV testing rate after 12 months. Generalized linear mixed models (GLMM) will be used for the analysis. Intervention status and time will be considered fixed effects, while sites and individual participants with multiple measurements across the four follow-ups will be considered random effects.

The secondary outcomes will include a series of binary variables (continuous variables will be categorized into binary variables), such as number of sexual partners, frequency of anal sex, sex without condoms, frequency of HIV testing (including both facility-based and selftesting), awareness of syphilis status, social network engagement, and others. These will be analyzed similar with the primary outcomes. Furthermore, the interaction effect of intervention between individual level and community level will also be analyzed.

After the 2nd follow-up, we will conduct an interim analysis to evaluate the effectiveness of digital images and videos. After the 4th follow-up, we will also evaluate the effectiveness of digital media and self-test kit using data collected from the 3rd and 4th follow-ups.

The data cleaning, sorting, and analysis will be carried out by team members who do not participate in the randomization and city administration and do not know the treatment allocation. Data analysts were not allowed to modify or delete data. If an outcome is missing for $<15 \%$ of participants, analyses will use a complete-case approach. Otherwise, multiple imputation will be used. Participants who miss the follow-up will be included and assumed not achieve the primary and secondary outcomes during the missed follow-up period. 
We will also carry out a subgroup analysis based on age ( $>30$ years old versus $\leq 30$ years old) and way of HIV testing (facility-based versus self-testing).

In order to measure contamination, we will conduct per protocol sub analyses to measure whether our results have been affected by contamination.

Results of this study will be distributed to target cities and national stakeholders.

\section{Adverse events and others}

We will capture potential unexpected adverse events via spontaneous self-report. We build one WeChat account for each city. Participants can contact us on WeChat so we can capture questions and potential unexpected adverse events. Additionally, some participants will be recruited through local CBOs. The respective $\mathrm{CBO}$ leaders can also help us to collect adverse events.

Adverse events will be fully and truly reported on a regular basis to the study PI.

If any modifications to the protocol are required at any point over the course of the trial, these changes will be communicated to relevant parties by email.

\section{Discussion}

Digital crowdsourcing approach has the potential to reach marginalized populations who face multi-level barriers to care, lower the cost of intervention development, aggregate community wisdom, and spur innovation [20, $26,29,31$. Several studies and reviews showed that the digital crowdsourcing can improve HIV services for MSM, promote HIV test uptake, and link men to care $[19,24,29]$. It can be a powerful vehicle for increasing HIV testing uptake and making messages more feasible to implement among MSM. The effectiveness of digital crowdsourcing has been examined in few randomized controlled trials, suggesting the need for more rigorous evaluation. We believe that a large-scale, multi-site cluster RCT is needed to evaluate the effectiveness of digital crowdsourcing.

There are several limitations that should be considered in this study. First, digital approaches may not be able to reach people who do not use online tools frequently. The CBOs in eleven target cities will also assist with recruitment, which may alleviate this problem to some extent. Second, all the information will be self-reported, introducing the potential for bias. However, our online survey allows a high degree of anonymity, we will not collect personal information like name or address, and we will highlight privacy protection methods in the informed consent. In this way, these potential sources of bias may be reduced. Third, MSM will receive $50 \mathrm{RMB}$ when they complete one online questionnaire. Part of them may deliberately falsify their answers in order to obtain the incentive. To reduce this bias, we have established a strict review mechanism. When a questionnaire has been completed, we will check it first. If it has only one logical error, we will verify with the participant. When there is more than one logical error in the questionnaire, the participant will no longer be included in the study. Furthermore, we have an administrator for each city to ensure that recruitment and review work is done in an orderly manner. Triangulation with CDC surveillance site data can facilitate this validation. Fourth, participants from the intervention and control cities may potentially communicate with each other, which may cause study contamination. We will use WeChat and Sojump Survey Software to avoid contamination. Participants will not know the city assignment. Participants will be required to add us as contacts on WeChat so that we can establish an online cohort. WeChat is a closed social app, if you want to talk to someone on WeChat, you have to add him/her as contact first. We will build $10 \mathrm{WeChat}$ accounts for the 10 cities so that participants from different cities cannot contact each other. The Sojump Survey Software was used to collect online questionnaire. In this software, participants will not receive any images or videos when they choose the control arm cites. Furthermore, if a participant did not choose the city he actually lives in, we can find his IP address through the Sojump Software. If his IP address is not consistent with his choice in the questionnaire, the participant will not be included in the study. Although the randomization of this trial is at city level, participants will not be aware of whether they are in the intervention group or the control group. Participants will also be asked whether they have seen the pictures or words which should only been sent to the intervention group in the last follow-up questionnaire.

We anticipate that this digital crowdsourcing intervention will increase HIV testing rate and promote safe sexual behaviors among MSM in China. The study outcomes will help to identify the effectiveness of the intervention. The intervention content can be accessed for free to the public. If successful, we hope that the health institutions will utilize this program as a resource and applied to the HIV prevention or to solve other health issues.

\section{Trial status}

This protocol was registered in chictr.org.cn with ID ChiCTR1900024350, identifier http://www.chictr.org.cn/ showproj.aspx?proj=36718. Protocol Version: 4.0, date: 27. March 2020. CONSORT Extension for Cluster Trials 2012 Statement was used to guide the writing of this protocol [34]. Recruitment started on 6 August 2019 and finished on 25 January 2020. At the time of this draft, the recruitment has completed. The original study sites included eight cities. Because we found the progress 
was slow in several cities during the recruitment, especially in the less developed areas, after waiting a reasonable amount of time and discussing with the advisory committee, we decided to add another three cities in our study. City E and City I are two independent cities which are assigned to one block according to the city's number of HIV-positive in 2018, average GDP in 2017, cumulative number of HIV-positive from 2012 to 2018, and year's population at the end of 2017. Random numbers were generated using SAS 9.4 software. Finally, City E was assigned to the control arm, City I was assigned to the intervention arm by randomization. City $\mathrm{K}$ is a city nearby City J; in this study, City J and City $\mathrm{K}$ are in the same cluster considering the number of estimated MSM in both cities is small. In other words, City J and City K were considered as one city in this trial. After adding three cities to the study, we have a total of ten clusters, five in the intervention group and five in the control group. After re-calculating sample size, the total sample size is 500 men (50 to each cluster).

\section{Supplementary information}

The online version contains supplementary material available at https://doi. org/10.1186/s13063-020-04860-8.

\footnotetext{
Additional file 1. Online survey instrument (English version)

Additional file 2. CDC surveillance survey instrument

Additional file 3. Intervention materials

Additional file 4. Table for secondary outcomes
}

Additional file 5. SPIRIT 2013 Checklist: Recommended items to address in a clinical trial protocol and related documents

\section{Abbreviations}

AIDS: Acquired immune deficiency syndrome; CBO: Community-based organization; CDC: Chinese Center for Disease Control and Prevention; GLMM: Generalized linear mixed models; HIV: Human immunodeficiency virus; LMIC: Low-income and middle-income countries; MSM: Men who have sex with men; QR code: Quick response code; RCT: Randomized controlled trial; STI: Sexually transmitted infection; UNAIDS: The Joint United Nations Programme on HIV and AIDS

\section{Acknowledgements}

We would like to thank all study participants and staff members at Blued, local CBOs, and Shandong Center for Disease Control and Prevention who contributed.

\section{Authors' contributions}

WM, JT, WT, and CR conceived the study. DK, XT, ML, and GW participated in building the surveillance sentinel. $M L, C R, K J, Z X, Z Z, Y L, C L, L W, Y Y$, and $Y L$ participated in MSM recruitment. The manuscript was prepared by $C R$ and was carefully revised and edited by WM, DK, JT, and WT. All authors read and approved the final manuscript. All decisions about authorship follow conventions established by the International Committee of Medical Journal Editors.

\section{Funding}

This work was supported by the National Key Research and Development Program of China (2017YFE0103800), National Institutes of Health (NIAID 1R01Al114310-01), NIMH (R34MH109359 and R34MH119963), and The National Nature Science Foundation of China (81903371). The listed funders had no role in any step of this study.

\section{Availability of data and materials}

Data is not applicable now. Materials can be found in Additional file 3. People who are interested in the data can contact us after the trial is completed.

\section{Ethics approval and consent to participate}

This protocol has been developed in accordance to the SPIRIT Checklist (see Additional file 5). Institutional Review Board (IRB) approval has been obtained from School of Public Health, Shandong University on 6 March 2019 (20190210). All participants will provide consent prior to taking part in the study.

\section{Consent for publication}

Not applicable.

\section{Competing interests}

The authors declare that they have no competing interests.

\section{Author details}

${ }^{1}$ Department of Epidemiology, School of Public Health, Cheeloo College of Medicine, Shandong University, Jinan 250012, Shandong, China. ${ }^{2}$ University of North Carolina Chapel Hill Project-China, No. 2 Lujing Road, Guangzhou 510095, China. ${ }^{3}$ Institution for AIDS/STD Control and Prevention, Shandong Center for Disease Control and Prevention, Jinan 250014, Shandong, China.

Received: 11 May 2020 Accepted: 1 November 2020

Published online: 17 November 2020

\section{References}

1. The progress in AIDS prevention and control in China, 2019. National Health Commission of the People's Republic of China. http://www.nhc gov.cn/jkj/s3586/201911/c2388ce70bdd404ea6dfcd886591784d.shtml. Accessed 7 Mar 2020

2. National Center for AIDS/STD Control and Prevention, China CDC. Update on the AIDS/STD epidemic in China and main response in control and prevention in 2011. Chin J AIDS. 2012;18(02):64.

3. Chow E, Wilson DP, Zhang L. The rate of HIV testing is increasing among men who have sex with men in China. HIV Med. 2012;13(5):255-63.

4. Zou H, Hu N, Xin Q, Beck J. HIV testing among men who have sex with men in China: a systematic review and meta-analysis. AIDS Behav. 2012; 16(7):1717-28.

5. Joint UN Programme on HIV/AIDS (UNAIDS). 90-90-90-an ambitious treatment target to help end the AIDS epidemic. UNAIDS. 2014 http://www.unaids.org/en/resources/documents/2017/90-90-90. Accessed 16 Dec 2019

6. Garett R, Smith J, Young SD. A review of social media technologies across the global HIV care continuum. Curr Opin Psychol. 2016:9:56-66.

7. Taggart T, Grewe ME, Conserve DF, Gliwa C, Roman Isler M. Social media and HIV: a systematic review of uses of social media in HIV communication. J Med Internet Res. 2015;17(11):e248.

8. Schnall R, Travers J, Rojas M, Carballo-Dieguez A. eHealth interventions for HIV prevention in high-risk men who have sex with men: a systematic review. J Med Internet Res. 2014;16(5):e134.

9. Muessig KE, Nekkanti M, Bauermeister J, Bull S, Hightow-Weidman LB. A systematic review of recent smartphone, internet and web 2.0 interventions to address the HIV continuum of care. Curr HIV-AIDS Rep. 2015;12(1):173-90.

10. Tso LS, Tang W, Li H, Yan HY, Tucker JD. Social media interventions to prevent HIV: a review of interventions and methodological considerations. Curr Opin Psychol. 2016;9(4):6-10.

11. Tucker JD, Muessig KE, Cui R, Bien CH, Lo EJ, Lee R, et al. Organizational characteristics of HIV/syphilis testing services for men who have sex with men in South China: a social entrepreneurship analysis and implications for creating sustainable service models. BMC Infect Dis. 2014;14(1):601.

12. Cao B, Saha PT, Leuba SI, Lu H, Tang W, Wu D, et al. Recalling, sharing and participating in a social media intervention promoting HIV testing: a longitudinal analysis of HIV testing among MSM in China. AIDS Behav. 2019; 23(5):1240-9.

13. Guo $Y$, Li X, Fang $X$, Lin $X$, Song $Y$, Jiang $S$, et al. A comparison of four sampling methods among men having sex with men in China: implications for HIV/STD surveillance and prevention. AIDS Care. 2011a;23(11):1400-9. 
14. Guo Y, Li X, Stanton B. HIV-related behavioral studies of men who have sex with men in China: a systematic review and recommendations for future research. AIDS Behav. 2011b;15(3):521-34.

15. Wu Z, Xu J, Liu E, Mao Y, Xiao Y, Sun X, et al. HIV and syphilis prevalence among men who have sex with men: a cross-sectional survey of 61 cities in China. Clin Infect Dis. 2013;57(2):298-309.

16. Liu M, Ma B, Mi G. A comparison of web-based survey and national sentinel surveillance among MSM in China. Chin J AIDS STD. 2016;22(06):441-3.

17. Cao B, Gupta S, Wang J, Hightow-Weidman LB, Muessig KE, Tang W, et al. Social media interventions to promote HIV testing, linkage, adherence, and retention: systematic review and meta-analysis. J Med Internet Res. 2017; 19(11):e394.

18. Young SD. Recommended guidelines on using social networking technologies for HIV prevention research. AIDS Behav. 2012;16(7):1743-5.

19. Tucker JD, Fenton KA. From the public, for the public: innovation challenge contests to enhance HIV responses. The Lancet HIV. 2018;5(3):e113-5.

20. Tang W, Ritchwood TD, Wu D, Ong JJ, Wei C, Iwelunmor J, et al. Crowdsourcing to improve HIV and sexual health outcomes: a scoping review. Curr HIV-AIDS Rep. 2019;16(4):270-8.

21. Tucker JD, Day S, Tang W, Bayus B. Crowdsourcing in medical research: concepts and applications. PeerJ. 2019;7:e6762.

22. Lee YJ, Arida JA, Donovan HS. The application of crowdsourcing approaches to cancer research: a systematic review. Cancer Med. 2017;6(11): 2595-605.

23. Mavandadi S, Dimitrov S, Feng S, Yu F, Sikora U, Yaglidere O, et al. Distributed medical image analysis and diagnosis through crowd-sourced games: a malaria case study. PLOS ONE. 2012;7(5):e37245.

24. Tucker JD. Crowdsourcing HIV testing: a pragmatic, non-inferiority randomized controlled trial in china. Am J Trop Med Hyg. 2015;93S(4):187-8.

25. Wazny K. Crowdsourcing's ten years in: a review. J Glob Health. 2017;7(2).

26. Tucker JD. Crowdsourcing to promote HIV testing among MSM in China: study protocol for a stepped wedge randomized controlled trial. Trials. 2017:18(1).

27. Khare R, Good BM, Leaman R, Su Al, Lu Z. Crowdsourcing in biomedicine: challenges and opportunities. Brief Bioinform. 2016;17(1):23-32.

28. Crequit P, Mansouri G, Benchoufi M, Vivot A, Ravaud P. Mapping of crowdsourcing in health: systematic review. J Med Internet Res. 2018;20(5):e187.

29. Tang W, Wei C, Cao B, Wu D, Li KT, Lu H, et al. Crowdsourcing to expand HIV testing among men who have sex with men in China: a closed cohort stepped wedge cluster randomized controlled trial. PLOS Med. 2018;15(8): e1002645.

30. Deal SB, Lendvay TS, Haque MI, Brand T, Comstock B, Warren J, et al. Crowd-sourced assessment of technical skills: an opportunity for improvement in the assessment of laparoscopic surgical skills. Am J Surg. 2016;211(2):398-404

31. Wazny K. Applications of crowdsourcing in health: an overview. J Glob Health. 2018;8(1)

32. Wu D, Huang W, Zhao P, Li C, Cao B, Wang Y, et al. A crowdsourced physician finder prototype platform for men who have sex with men in China: qualitative study of acceptability and feasibility. JMIR Public Health Surveill. 2019;5(4):e13027.

33. Tucker JD, Tang W, Li H, Liu C, Fu R, Tang S, et al. Crowdsourcing designathon: a new model for multisectoral collaboration. BMJ Innovations. 2018;4(2):46

34. Campbell MK, Piaggio G, Elbourne DR, Altman DG, CONSORT Group. Consort 2010 statement: extension to cluster randomised trials. BMJ. 2012; 345:e5661

\section{Publisher's Note}

Springer Nature remains neutral with regard to jurisdictional claims in published maps and institutional affiliations.

Ready to submit your research? Choose BMC and benefit from:

- fast, convenient online submission

- thorough peer review by experienced researchers in your field

- rapid publication on acceptance

- support for research data, including large and complex data types

- gold Open Access which fosters wider collaboration and increased citations

- maximum visibility for your research: over $100 \mathrm{M}$ website views per year

At $\mathrm{BMC}$, research is always in progress.

Learn more biomedcentral.com/submissions 